\title{
HARTMUT BöHME \\ Vergangenheit und Zukunft im Traum. Zur Traumhermeneutik bei Artemidor von Daldis und Ludwig Binswanger.
}

"...denn auf den langen Traum des Lebens sind die kleinen bunten Träume der Nacht wie Phantasieblumen gestickt und gezeichnet." (Jean Paul) ${ }^{1}$

"Eines Schattens Traum, der Mensch." (Pindar: Achte Pythische Ode, V. 95f)

"Zwischen ,es träumte mir' und ,ich träume' liegen die Weltalter. Aber was ist wahrer? So wenig die Geister den Traum senden, so wenig ist es das Ich, das träumt."

(Theodor W. Adorno: Minima Moralia) ${ }^{2}$

\section{Heraklit und die Folgen}

"Herakleitos sagt, dass die Wachenden ein und dieselbe gemeinsame Welt (éna kai koinòn kosmon) haben, während von den Schlafenden ein jeder sich zu seiner eigenen (Welt; eis ídion apostréphesthai) abwende." (DK 22 B 89) 3 Diese, eigene' Welt des Schlafenden heißt ídios kósmos, die Welt der Träume. "In der Nacht entzündet der Mensch ein Licht für sich selbst, sterbend, seine Sehkraft ist erloschen." (DK 22 B 26). - Heraklit (535 - ca. 475 v. u.Z.) trifft die Überzeugung der gesamten antiken Kultur, dass der Wachzustand eine Welt des Zugänglichen, des Lichterfüllten, die Welt also des Öffentlichen, des Gemeinsamen und des Logos darstelle: koinos kósmos. Im Traum tauchen wir in eine geschlossene, idiosynkratische Welt ein, die auch das Private, mit niemandem Geteilte darstellt. Bei Heraklit ist allerdings charakteristisch, dass er der erste Philosoph war, der dem Logos Universalität zusprach. Dies hieß aber auch, dass der Logos wie den Tag so auch die Nacht und den Traum durchherrscht. Im Fragment DK 22 B 72 ist die Rede vom "Logos, der das All" verwaltet. Oder es heißt: „Der Gott ist Tag-

1 Jean Paul: Leben des vergnügten Schulmeisterlein Maria Wutz in Auenthal. In: Jean Paul: Sämtliche Werke. Hg. v. Norbert Miller. München 1960ff, hier Abt.I, Bd. I, S. 460 .

2 Theodor W. Adorno: Minima Moralia. Frankfurt/M. 1985, S. 252.

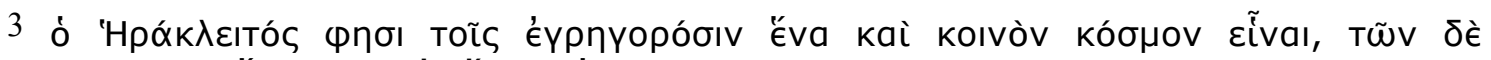

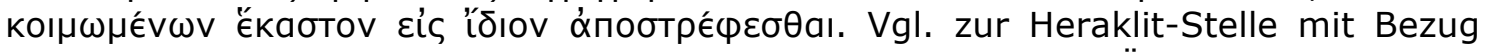
auf Binswanger: Lenk, Elisabeth: Die unbewußte Gesellschaft. Über die mimetische Grundstruktur in der Literatur und im Traum; München 1983, S. 152-58. 
Nacht, Winter-Sommer, Krieg-Frieden, Sättigung-Hunger - alle Gegensätze" (DK 22 B 67). Oder: "Dasselbe ist: lebendig und tot und wach und schlafend und jung und alt. Denn dieses ist umschlagend in jenes und jenes umschlagend in dieses." (DK 22 B 88) Überall herrschen Gegensätze, sie bezeichnen die Welt der Erscheinungen; aber der tieferen Einsicht erschließt sich die Fundamentalität des einen Logos, der die Gegensätze in sich vereint.

Man soll die Reichweite dieser Auffassung Heraklits nicht unterschätzen: noch heute sind die Human- und Sozialwissenschaften ,Tageswissenschaften'4: sie beziehen sich auf die geteilte, öffentliche Welt, in der und über die, allgemeine Aussagen' möglich sind, in der man Indentifizierungen von Sachverhalten vornehmen kann und regelhafte, politisch wie moralisch wie physisch verbindliche Verknüpfungen zwischen den Elementen des Tagesfeldes möglich sind. Der Tag ist die rationalisierbare Welt, des Realen und Identischen, wovon ausweisbares Wissen möglich ist. Von der Nacht und ihren Gesichten können wir vielleicht Erfahrungen haben, aber kein Wissen und vor allem keine Gemeinsamkeit. Es ist darum kein Zufall, dass die Traumdeutung - wenn sie nicht in der Obhut von Priestern lag und Arkanwissen war - im Abendland durchschnittlich zum okkulten Bereich zählte. Man kennt die abfälligen Äußerungen Platons über den Traum in der "Politeia" (Pol. X, 571c-d). Von Träumen und Gesichten Kenntnisse zu haben, stand immer in Zwielicht: ist dies ein extraordinäres Einweihungswissen oder eine dubiose, womöglich dämonische Kunde von Kräften, auf die sich einzulassen nicht ratsam ist. Die Romantik, als Komplement zu der im Zeichen des Tages und des Lichtes stehenden Aufklärung, war vielleicht die erste Epoche, die entschieden und positiv die Signatur der Nacht, des Dunkels, des Traums und der Phantasie - und damit auch des Unbewussten -, affirmierte. 5

Damit war die Möglichkeit eingeräumt, dass die Vernunft als diejenige Instanz auftrat, welche die, andere Logik' des Traums als die bloß entstellte Form ihrer eigenen Logik darstellte. Gerade als Vernunft konnte der Logos die Deutungshoheit in der Sphäre des

4 Vgl. dazu Bretthauer, Bastian: Die Nachtstadt. Tableaus aus dem dunklen Berlin; Frankfurt am Main 1999.

5 Heise, Jens: Traumdiskurse. Die Träume der Philosophie und die Psychologie des Traums. Frankfurt/M. 1989, S. 182-218. 
Traums beanspruchen. Diese Überzeugung reicht hin bis zur aufklärende Hermeneutik des Traums bei Freud. Logos in der Antike ist nicht nur Vernunft, sondern auch Rede, Sprache und Ordnung. Wenn auch der Traum dem Logos angehört, welcher am Tage herrscht, dann heißt dies: der Traum ist eine andere Sprache, ídios logos vielleicht, aber doch logos. Wenn es - wie Jens Heise in seinem anregenden Buch "Traumdiskurse" annimmt - die große Leistung Freuds sei, dass er den linguistic turn in der Traumtheorie herbeigeführt habe, eine Wendung, den Jacques Lacan dann vollendet habe, dann muss schon Heraklit als der Urgroßvater dieser These von der Sprachlichkeit oder wenigstens von der Sprachähnlichkeit der Träume gelten. Freilich: der Traum ist eine "Rhapsodie der Bilder" und das „Bild ist ein Sprechen, das nicht formuliert", so dass man es durchaus eine Schwäche aller Traumtheorien von Freud bis Lacan und Heise nennen kann, dass sie den "Traum nur als Rede aufgefasst" und eine "Grammatik der Bildhaftigkeit" nicht hinlänglich ausgearbeitet haben. ${ }^{6}$

Den heraklitschen Gegensatz von koinos und ídios kósmos darf man auch als Gegensatz von ,öffentlich' und ,privat' auslegen. Die Agentien der Seele begründen im Schlaf eine geschlossene Privatwelt. Diese clôture, die Schließung des Traums, wird von Freud in der "Traumdeutung" durch Übersetzung der idiosynkratischen Privatsprache des Traums in den Diskurs der Traumgedanken und ihrer Deutung gleichsam geöffnet und befreit: aus dem stummen Spiel der Rätselbilder wird die klare Rede, welche der Traum aus seiner Abgewandtheit zurückholt in die, gemeinsame Welt'. Diese Auffassung herrschte lange vor Freud und noch lange über ihn hinaus, z.B. wenn der Jungianer Ernst Aeppli in seinem Buch "Der Traum und seine Deutung" schreibt: "Das Erlebnis des Traumes gehört zu den persönlichsten, sich wiederholenden Erfahrungen des Menschen. Er und kein anderer träumt seine Träume, erlebt dies oft fremdartige Nachtgeschehen. Fremdartig, weil es ohne sein Zutun sich ereignet und sich abspielt in einer Welt, die gar nicht die inm vertraute Welt des Tages ist."7 Aeppli wiederholt die Heraklitsche

6 Michel Foucault: Einleitung. In: Binswanger, Ludwig: Traum und Existenz. Aus dem Französischen und mit einem Nachwort von Walter Seitter; Bern - Berlin 1992, S. 16-20, 32.

7 Aeppli, Ernst: Der Traum und seine Deutung; 2. Aufl. Zürich 1943, S. 13. 
Einsicht in anderen Worten - und gibt zu erkennen, dass hier ganz vom Tagesbewusstsein aus gedacht wird. Nur diesem nämlich erscheint der Traum fremdartig, während es gerade zu den Merkmalen des Traumes gehört, dass, in ihn eingetaucht, dem Träumenden alles in keiner geringeren Selbstverständlichkeit widerfährt als die kompakte Realität des Tages. Vom Traum aus gesehen ist die Tagesrealität das Fremde.

Zu Recht betont Aeppli indes das Unwillkürliche im Traum. Ihm ist wesentlich, dass er für Intentionen verschlossen bleibt, während jede noch so massive Realität der Tagwelt niemals die in ihr gefangenen Akteure daran hindert, ihre Intentionen und Handlungen gezielt zur Veränderung dieses kompakten Seins einzusetzen. Deutlich wird, dass bei Aeppli der Traum als Negativum erschient (Abwendung, fremdartig, unwillkürlich, nicht vertraut, eigentümlich). Die Tagwelt ist das Positivum. Die Beraubung, das Privative des Traums ist eben das, was inn zum radikal Eigensten macht: das Ich kann, im Amt' vertreten werden, als Redner oder Sachbearbeiter, vielleicht sogar als Familienvater oder Liebhaber, aber nicht als Träumer. Das Ich-Sein, das sich auf das cartesische Cogito gründet, ist gerade nicht das Sein, worin ich ,kein anderer' und deswegen unaustauschbar - also ídios kósmos - bin; sondern dem Cogito der Tagwelt emergiert (ergo sum) ein Sein des Allgemeinen und Öffentlichen, worin das Subjekt gegen alle anderen Subjekte sich austauschen kann. Dadurch erst wird die gemeinsame Welt der Gesellschaft konstituiert, aber nicht das Sein, das ich gegen niemanden tauschen kann. So kann man argumentieren, dass es gerade das Träumen ist, das die Jeweiligkeit des eigenen Seins anzeigt und hervorbringt (somnio, ergo sum), so dass die privative imaginatio des Traums eher als die cogitatio der Akt wäre, aus dem das Sein des je eigenen Ich emergiert.

Aber auch das ist nur ein Schein, nämlich dann, wenn der Traum etwas ganz und gar Überpersönliches anzeigt: die Sprache der Götter; aber auch dann, wenn er, wie bei C.G. Jung und Aeppli, zur Darstellung gattungsallgemeiner Archetypen wird. Was ist der Traum? Wer träumt inn? Wer gibt ihn oder wer hat inn? Wo findet er statt? Welchen ontologischen Status hat er? Nicht ohne Grund mahnt Adorno: "Zwischen ,es träumte mir' und ,ich träume' liegen die Weltalter. Aber was ist wahrer? So wenig die Geister den Traum 
senden, so wenig ist es das Ich, das träumt."8 So kann man die Frage Lichtenbergs, was es wäre, was in mir denkt ${ }^{9}$, abwandeln: Was ist es, was in mir träumt? Die Götter? Die Wünsche? Das Unbewusste? Die Archetypen? Die mit sich selbst spielende Einbildungskraft? Die Vergangenheit? Die Zukunft?

Das Unaustauschbare und Nicht-Vertretbare des Traums lässt also den Spruch Heraklits auch anders auslegbar: der ídios kósmos begründet eine Sphäre, in der das Nächste und das Fernste, von denen wir nicht wissen, wie sie uns angehen, ihren Raum finden: nirgends, wenn nicht hier. Dieser Gedanke erlaubt eine andere Deutung der Nachtgesichte als diejenige Freuds zu entwickeln, der nach dem Muster der Verdrängung aus den Träumen die Zeichen des Verdrängten abliest. Diese andere Deutung führt einerseits in die Traumdeutung der Antike zurück, andererseits zur Auffassung des jungen Foucault, wie er sie in Auseinandersetzung mit Ludwig Binswanger entwickelt. Worum geht es dabei?

"Der Traum", so Aeppli, „ist ein Hüter der Tradition."10 Dies ist eine Auffassung, die für Freud und C.G. Jung, trotz aller Differenzen, gemeinsam gilt. Der Traum wird wesentlich durch seine Referenz auf Vergangenheit bestimmt, die biographische Vergangenheit des Träumenden oder die archetypische Vergangenheit der Menschheit. Hierbei wird der Traum wesentlich mit der (unbewussten) memoria fusioniert. Der Traum als unbewusste Erinnerung bringt das Unabgegoltene des Vergangenen ins Spiel; er inszeniert und gestaltet es; er ist die Darstellungsform des Vergangenen, von dem das Tagesbewusstsein nichts weiß. Darum kann man sagen, dass der Traum die Tradition hütet. Als Hüter ist er sowohl Bewahrer wie Wächter von etwas, was da war und nicht wiederkehren soll - und doch wiederkehrt, weil es zugleich den Wunsch und das Verbot des Wunsches enthält (Freud); oder von etwas, was unter den

\footnotetext{
8 Theodor W. Adorno: Minima Moralia. Frankfurt/. 1985, S. 252.

9 "Wir werden uns gewisser Vorstellungen bewußt, die nicht von uns abhängen; andere glauben, wir wenigstens hingen von uns ab; wo ist die Grenze? Wir kennen nur allein die Existenz unserer Empfindungen, Vorstellungen und Gedanken. Es denkt, sollte man sagen, so wie man sagt: es blitzt. Zu sagen cogito, ist schon zu viel, so bald man es durch Ich denke übersetzt. Das Ich anzunehmen, zu postulieren, ist praktisches Bedürfnis." (Lichtenberg, Georg Christoph: Schriften und Briefe; hg. v. Wolfgang Promies. 4 Bde. Frankfurt am Main 1992, hier: Bd. II, S.- 412, Sudelbücher K 76).
}

10 Aeppli (wie Anm. 7), S. 30) 
Entfremdungen des Tages vergessen ist und da sein sollte, weil es bewahrenswert ist und darum behütet werden muss (C.G. Jung).

Die , andere' Möglichkeit der Auslegung des heraklitischen Spruchs wird von Foucault betont: "Was den ídios kósmos des Träumers konstituiert, ist nicht die Abwesenheit von Wahrnehmungsinhalten, sondern deren Ausarbeitung zu einem isolierten Universum. Die Tramwelt ist eine eigene Welt: nicht in dem Sinn, dass sich in ihr die subjektive Erfahrung über die Normen der Objektivität hinwegsetzt" dies wäre die Freudsche Deutung -, "sondern in dem Sinn, dass sich eine Welt konstituiert, die ursprünglich meine ist, indem sie mir meine eigenste Einsamkeit kundtut." 11 - "Die Kosmogonie des Träumens ist der Ursprung der Existenz selbst."12 Somnio, ergo sum.

Der junge Foucault, der noch deutlich in der Nähe zur Existenzphilosophie steht, legt das, was Aeppli als das Unvertretbare des Träumers im Traum bezeichnete, radikal aus als die im Traum eröffnete Existenzialität des jemeinigen Ich, des Idiopathischen also, worin das Ich ganz und gar, nackt, bei sich ist und zugleich transzendiert durch den Kosmos, der ursprünglich meiner ist, ein Kosmos also, der ich selbst bin. Im Traum - und darin sind sich alle modernen Traumdeuter einig - ist niemand anwesend außer das Ich selbst.

Dieser Gedanke begründet die Verbindung von "Traum und Existenz", wie sie Foucault auf den Spuren Binswangers entfaltet. Die andere Weise nun, Träume nicht in den Bann des Vergangenen zu rücken, ist ihre Fähigkeit, ,Gesichte' zu bilden: ein Sehen also in die Zukunft, ein "Fernsehen".13 Dies ist die übliche Auffassung der Antike. Wir finden sie indes auch in anderen Religionen, etwa der jüdischen, wenn Propheten - wie Jeremia, Daniel oder auch Joseph Traumgesichte zuteil werden, welche als göttliche Offenbarungen des Zukünftigen gelten. Dass im Traum sich die Götter mitteilen und zwar so, dass daraus die Zukunft abgelesen werden kann, ist Gemeingut der griechischen und römischen Traumexperten. Träume transzendieren die Gegenwart des Träumers nicht im Modus der Memoria, sondern im Modus der Imaginatio (phantasia). Mit welchem dieser beiden Vermögen man den Traum mehr kooperieren lässt -

11 Foucault (wie Anm.6), S. 46.

12 Ebd. S. 47.

13 Ebd. S. 42. 
mehr mit der memoria oder mehr mit der imaginatio -, dies entscheidet über den Zeitmodus, welcher für den Traum leitend ist. Man erkennt, dass das zur Zukunft hin offene Traumgesicht mit dem existenziellen Sinn, wie er von Binswanger und Foucault angedeutet ist, jedenfalls das gemeinsam hat, dass die Traumbilder irreduzibel (nicht auf Vergangenheit rückführbar) sind, sondern eine eigene Wucht hin in die Zukunft entwickeln. Das Irreduzible des Traums heißt, dass er nicht als Rekombination von Elementen der Vergangenheit, die im Gedächtnis abgelegt sind, bestimmt werden kann, als Rebus unbewusster Verdrängungen. Wenn Foucault dem Traum das Moment der Schließung (clôture) zuspricht, durch das die Subjektivität des Subjekts sich als radikal eigener Kosmos bildet, dann ergibt sich daraus ein Gegensatz zu den Träumen, die Zukunft eröffnen. In diesen wird das Ich des Traums in der Weise transzendiert, dass es eben das Ich nicht ist, das der Autor des Traums wäre, sondern die Götter: dadurch erst wird der Traum zum Orakel, zu einer ebenso verrätselten wie eindeutigen, objektiven Eingabe. Hier wird die Imaginatio des Schläfers zum Medium der Götter, die im Traum die Zukunft zugleich bezeichnen wie verhängen. Bei den Traumgesichten sind die Traumzeichen deswegen nicht geordnet zu einer Erzählung aus der Geschichte des Träumers; sondern sie bilden den Prätext seiner Zukunft, der zugleich ein Präskript, eine Vorschrift enthält. Eine solche Traum-Konzeption rechnet - so Foucault - mit der "Möglichkeit, dass die Wahrheit der Welt sich selber voraussehen und ihre Zukunft in einem allerdings nur wirren Bild einholen kann."14 Das ist das Transzendierende des Traums. Die imaginatio ist das Medium dieser Transzendierung.

"Niemand", so Spinoza, "hat die Offenbarung Gottes anders als mit Hilfe der Imagination empfangen." 15 Das von allen irdischen Bestimmungen freieste Vermögen, die Einbildungskraft, ist also das geeignetste Medium, um zur Darstellung des absolut Objektiven, nämlich Gottes zu werden. Gerade das Unwillkürliche des Traums

14 Ebd. 39.

15 Spinoza, Benedict Baruch: Theologisch-politische Abhandlung (Tractatus theologico-politicus, zuerst 1670). Übers. u. hg. v. J.H. Kirchmann. Berlin 1870, S. 17 (= Kap. I,52), zitiert bei Foucault (wie Anm. 6), S. 35. (Die korrekte wörtliche Übersetzung lautet: So können wir zusammenfassen, dass niemand außer Christus göttliche Offenbarungen ohne die Hilfe der imaginatio empfangen hat, weder in Worten noch in Bildern.) 
macht ihn zum empfindlichen Membran für alle Offenbarungen. Sie kommen jenseits des Bewusstseins zur Darstellung. Wie bei den antiken Traumdeutern, die in den Träumen die Zeichen der Götter lesen, ist es auch bei Spinoza der Offenbarungscharakter des Traums, der zur sorgfältigen Unterscheidung verpflichtet, ob es sich um Träume handelt, die nur zufälligen Reizen des Körpers folgen, oder um solche, in denen die Imagination zur Membran Gottes wird. Bebildert der Traum die Launen der Organe, so bleibt er ídios kósmos: der Körper regt im Schlaf die Einbildungskraft zu allerlei Possen auf, die ein täuschendes Bilder-Spiel aufführen. Wenn die Eingeweide, die Geschlechtsteile, das Herz oder der Magen des nachts ihre Aktivitäten auf die Imagination ausdehnen, wird der Traum zur Blähung der Innereien. Diese Traumsorte muss man umso sorgsamer von den offenbarenden Gesichten unterscheiden, als diese den absoluten Gegenpol zum Vexierspiel der Organe bilden - und doch sehen sich die Träume so ähnlich!

Die Wahrheit der pronoetischen Träume kann ebenso das Individuum betreffen wie etwa den Ausgang der bevorstehenden Schlacht: entscheidend ist, dass die Traumzeichen hier Objektivität stiften, weil sie die Eingaben der zukunftskundigen Mächte sind. Die imaginatio ist das wertvolle Vermögen, durch das der Mensch zum Empfängnisorgan von Sendungen der Götter wird. Die Götter schicken Bilder. Die Traumdeuter fertigen daraus ein Transskript der verschlüsselten Botschaften. Was sie dafür benötigen ist zumindest ein Lexikon. Innerhalb der Traumgattung sind es also die Traumgesichte, welche die für das Abendland so einflussreiche Traumdeutungsform der lexikalisch-enzyklopädischen Traumbücher begründet haben. Um diesen Typ soll es im Folgenden gehen.

\section{Die „Oneirokritika" des Artemidor von Daldis}

Wie immer der Streit über die Bild- oder Sprachlogik des Traums ausgehen mag - zwischen modernen psychologischen Auffassungen, nach denen der Traum aufs intimste mit gegenwärtigen und vergangenen Erfahrungen des Individuums zusammenhängen, und der antiken Auffassung des Traums liegen gewaltige Unterschiede. Wir werden sie an dem einzigen erhaltenen vollständigen Traumbuch der Antike entfalten. Die "Oneirokritika" (,Das Traumbuch') des Artemidor von Daldis stammen aus dem 2 . nachchristlichen 
Jahrhundert. ${ }^{16}$ Artemidor war professioneller Traumdeuter und Wahrsager, Anhänger der stoischen Schule. ,Óneiros' heißt Traum, Traumgesicht, Traumbild, aber auch die Nichtigkeit und leere Einbildung, gelegentlich auch der Traumgott selbst (hypnos; lat. somnium). "Enhypnion' ist ein weiteres Wort für Traumbild. 'Oneirokritikós', woraus der Titel der aus fünf Büchern bestehenden Abhandlung des Artemidor abgeleitet ist, meint, das die Traumdeutung betreffende'. Ein ,oneirokrítes' oder, oneirómantis' ist ein Traumdeuter. Schon in der Ilias (z.B. I, 63; II, 1-80; X, 496f) und in der Odyssee (IV, 795-841; VI, 13-49; XIV, 495; XIX, 535-556, 568) kommen Träume und Traumdeuter vor, die, neben dem Seher und dem Opferpriester, eine institutionelle Stellung einnehmen. Durch die gesamte Antike zieht sich die Beschäftigung mit Träumen, die als Eingabe von Göttern galten. Ihre professionelle Deutung stand als Gabe der Götter hoch im Ansehen. Die Antike war eine traumgläubige Epoche, wie Karl Brackertz schreibt. ${ }^{17}$ Das älteste Traumbuch stammt aus Ägypten und wird auf die 12. Dynastie datiert (2000-1700 v.u.Z.). Obwohl die Griechen die Gattung der Traumbücher fortführten, sind die Oneirokritika des Artemidor das einzige vollständige Zeugnis, das uns umfassende Einblicke in die Traumhermeneutik gewährt. Wie wir sehen werden, enthalten die Oneirokritika auch sehr viel über Volksgebräuche, Sitten und Glaubensvorstellungen der griechischen Kultur.

Die Verbindung des Traumkundigen zum Seher und Opferpriester zeigt zweierlei: Träume haben und Traumdeutung ihren Platz in hieratischen Kontexten; zweitens sind Träume - anders als bei Freud - chiffrierte Zukunft. Das Zukünftige wissen zu wollen, ist nicht nur ein alltagspraktisches Bedürfnis, sondern spielt eine erstrangige Rolle in religiösen und politischen Zusammenhängen. Im Traum äußert sich die göttliche Vorsehung (prónoia); diese zu erkennen erfordert erfahrene Experten. Durchaus im Einklang zum zitierten Fragment Heraklits heißt es bei Artemidor: "In jedem Fall ist auch der Träumende ein Teil des Alls." 18 Was sich im Traum zeigt, gehört zur

\footnotetext{
16 Vgl. dazu Heise (wie Anm. 5), S. 52-86; Näf, Beat: Traum und Traumndeutung im Altertum. Darmstadt 2004, S., 124-128. - Walde, Christine: Antike Traumdeutung und moderne Traumforschung. Düsseldorf 2001.

17 Artemidor von Ephesos: Das Traumbuch. Übers. und erl. v. Karl Brackertz. Zürich und München 1979, S. 349-391.

18 Ebs. S. 166 (Buch II, 36).
} 
Ordnung des Kosmos - oder zu dessen Störung. In jedem Fall ist es niemals gleichgültig. Weil der Traum von den Göttern geschickt ist, kommt ihm ein Höchstmaß an Bedeutung zu. Träume habe eine orientierende, Zukunft erschließende und handlungsrelevante Funktion; keineswegs sind sie Chiffren einer (womöglich verdrängten) Vergangenheit. Die semantische Überdeterminierung von Träumen, die Freud konstatiert, gilt schon in der Antike. Und da der Sinn von den Göttern stammt, kann man von einer ,objektiven Semantik' sprechen. Die Traumsignifikanten sind Elemente des künftigen Geschehens des Kosmos im Großen und im Kleinen. Niemals ist der Traum eine bloß subjektive Vorstellungswelt, in der private Phantasmen ihr sinniges oder unsinniges Spiel spielen. Eine solche Auffassung verbietet sich, wenn Träume die Medien göttlicher Botschaften sind, oft sogar von Zeus selbst. Sie gewinnen damit höchste Dignität. Dies gilt jedenfalls von den oneírata, während die enhypnia eine andere Traumgattung darstellen, nämlich auf triviale physiologische Ursachen und Körperreize zurückgehen und "daher für die Zukunftsdeutung belanglos" sind. 19

Als bezahlter Traumausleger beschäftigt sich Artemidor ausschließlich mit prognostischen oneírata, deren Zeichen nicht etwa Symptome schlechter Verdauung oder sexueller Erregungen sind, sondern omina, Vorzeichen und Vorbedeutungen darstellen. Dies gilt auch für die Dichter, die in ihre Tragödien öfters Träume einschalten - mit durchweg pronoetischer Funktion. Georges Devereux hat gezeigt, dass die Träume durchweg in ebenso psychologisch plausibler wie dramaturgisch wirksamer Weise ins Handlungsgeschehen der Tragödien eingebaut sind.20 Wir heute, Kinder der Aufklärung, kennen Omina kaum noch oder glauben nur vorbehaltlich oder spielerisch an solche. Doch noch Georg Büchner lässt Oberlin wie Lenz an solche futurische Traum-Ahnungen glauben und selbstverständlich findet Heinrich von Ofterdingen in Träumen seinen Lebensweg vorgezeichnet. Zukunftsorientierende Zeichen lassen wir indes nur noch in Form prognostischer Kalküle und rationaler Abwägungen zu: sie halten mit einiger Verlässlichkeit Erwartungshorizonte stabil und reduzieren zu unserer Entlastung

19 Ebd. S. 361.

20 Devereux, Georges: Träume in der griechischen Tragödie. Eine ethnopsychoanalytische Untersuchung; Frankfurt am Main 1982. 
Komplexität. Eben diese Funktion aber haben antike Traumdeuter, wenn sie Träume lesen. Es käme innen nicht in den Sinn, Omina als Indizes oder Spuren einer Vergangenheit zu entziffern, die Aufschlüsse über biographischen Sinn erlauben. Vielmehr finden wir den oneirischen Charakter von Zeichen in allen Praktiken, die auf das Wissen des Zukünftigen aus sind, sei's der Vogelflug, die Organe geopferter Tiere oder die Konstellation von Sternen. Wie Artemidor die Traumzeichen durch wissenschaftliche Prozeduren zu entschlüsseln sucht, so ist auch die Astrologie für mehrere Jahrtausende eine seriöse Kunst, um die zukunftserschließende Narrativität von Sternen zu enträtseln. Traumdeutungen oder Orakel sind Zukunftskonstruktionen, nicht hermetische Formen der Memoria. Darum ist die Traumdeutung eine hieratische Kunst. Wie die Sterne eine Himmelsschrift der Götter sind, Charaktere künftiger Geschicke, die in ihrem bedeutsamen Gang beschlossen sind, so enthalten auch das Ephemere des Traums, das Flüchtige des Vogelflugs, der Wurf des Losorakels Botschaften der Götter, Warnungen, Winke, Präskripte, Verheißungen und Verhängnisse, die vom kundigen Mantiker oder Traumexperten enträtselt werden.

Was die Träume als Zukunftsorakel tauglich macht, hat ausgerechnet der wissenschaftlichste unter den Philosophen, Aristoteles, gut erklärt. In seiner Schrift "De somno et vigilia" erläutert er das Zur-Ruhe-Kommen der Seele im nächtlichen Schlaf, wodurch sie von den "Umtrieben des Körpers"21 gelöst wird. Gewöhnlich sind Seele und Körper, und - durch Wahrnehmung und Handeln - Seele und Außenwelt so miteinander verflochten und verstrickt, dass dabei eine Art Seelenlärm entsteht, in welchem verloren geht, was an zarteren Bewegungen aus der kosmischen Welt nur ein leises Echo in der Seele findet. Im tiefen Schlaf hingegen tritt eine Stille ein, in der die Seele "für die feinsten Bewegungen der Welt empfindlich, für die feinsten Regungen empfänglich" wird.22 Gerade indem die Verhakung von Körper und Seele im Schlaf gelöst wird, taucht „in Träumen ... die von ihrem Körper gelöste Seele in den kosmos ein"23, sie wird zum Resonanzraum fernster Mitteilungen. Dann werden die feineren Schwingungen der sympatheia wirksam,

\footnotetext{
21 Foucault (wie Anm. 5), S. 39.

22 Ebd.

23 Ebd. S. 40.
} 
jenes sympathetische Prinzip, das wie ein Fluidum die Seele mit dem Kosmos in Korrespondenz setzt. Im Traum ereignet sich vermittels der fluidalen Sympathie ein Erkennen dessen, was von weit her, den Göttern, den Sternen, den scheinbar toten Dingen als Mitteilung ausgeht und was dem Träumenden, vermöchte er denn zu verstehen, die Zukunft zu enträtseln vermag. Darauf beruht das Überpersönliche der Traumeingebungen, wie es für die vor-psychoanalytische Auffassung des Traums typisch ist. Doch zumeist versteht der Träumende die mitgeteilten Botschaften nicht. Er benötigt angesichts der Verdunkelung seiner Zukunft einen Deuter, den oneirokrítes, dessen Künste denn auch so gutes Geld wert sind wie heute diejenigen des Therapeuten. Ein solcher war Artemidor, der offenbar so wenig an einem Heiligtum institutionalisiert war wie die Sophisten, Wanderärzte, Rhetoren feste Orte hatten. Ein weitgereister Mann, wie er von sich selbst behauptet, aus der stoischen Schule, welche die Lehre von den Sympathien als die universale Vermittlungsform des Kosmos und seiner inn durchströmenden Weltseele entwickelt hatte. Das bildet den Hintergrund seiner Traumlehre.

\section{Klassifikationen des Traums}

Als erstes unterscheidet Artemidor das Traumgesicht (óneiros), das die Zukunft enthüllt, vom anhypnion, dem Traum, der die Gegenwart anzeigt. Es sei kein Wunder, wenn der Liebhaber von seinem Lieblingsknaben, der Hungrige vom Essen, der Durstige vom Trinken, der von Angst Geplagte von der Angst, und einer, der den Magen überladen hat, vom Erbrechen und Ersticken träumt. "Daraus kann man erkennen, dass Träume, deren Grundlage Affekte bilden, nichts über die Zukunft aussagen, sondern nur an Zustände der Gegenwart erinnern." 24

Das ist die Banalität der Träume, der duplizierte Lärm der Gegenwart, die dumme Repetition der organischen Regungen. Es ist gut zu wissen, dass es solche Träume gibt, um sich für sie gerade nicht zu interessieren. Um die falsche Investition von Aufmerksamkeit zu vermeiden, ist es nach Artemidor ratsam, viel über den Träumenden zu wissen: das erleichtert die Aussonderung der enhypnia und befreit von deren tautologischer Aufdringlichkeit. Übrigens kennt noch Freud - wie alle vor ihm - diese somatischen

\footnotetext{
24 Artemidor (wie Anm.16), S. $9 f$.
} 
Quellen von Träumen, die den reinen Traumtext verderben. Diesen Traumtext unverfälscht herzustellen, ist das Ziel Artemidors.

Ferner unterscheidet Artemidor theorematische und allegorische Gesichte. Die ersteren zeigen unverhüllt an, was dem Träumenden widerfahren wird, während die allegorischen Träume dies nur "durch anderes anzeigen" und in "verhüllten Anspielungen" aussprechen. 25 Das angezeigte Zukünftige kann sich ebenso auf unmittelbar Bevorstehendes wie auf Langfristiges, in der Tiefe der Zukunft Wartendes beziehen.

Für den Ansatz Artemidors, der sich auf ein breites, heute verlorenes Schrifttum zu beziehen behauptet, ist es charakteristisch, dass er nicht etwa kasuistisch, sondern klassifikatorisch vorgeht. Von Artemidor bis zu Freud gilt die Überzeugung, dass man den Sinn von Träumen nicht theoretisch deduzieren kann. Die großen griechischen Tragiker Aischylos, Sophokles und Euripides verwenden Träume durchweg kasuistisch, indem sie die Träume dramaturgisch in den Handlungszusammenhang der Stücke sowie in die Seelenkontexte des Träumenden einbauen. Dadurch werden Träume zu wichtigen Kreuzungspunkten der Dramaturgie. Für Artemidor dagegen kommt es darauf an, über ein Instrumentarium zu verfügen, das Typus, Art und Klasse des Traums auszumachen erlaubt. Dies erlaubt eine erste Einkreisung des Sinns, um im zweiten Schritt jene Ebene zu erreichen, auf der typisierte, oft winzige Traumelemente (Signifikanten) auf die eine Seite einer Bedeutungsgleichung gestellt werden können, der auf der anderen Seite das Signifikat gegenübersteht (Element a ,bedeutet' b). Dies entspricht der Struktur des Lexikons. Dabei können, wie bei jedem Lexikon, einem Signifikanten mehrere Signifikate zugeordnet werden - und umgekehrt. So mag das Signifikat ,drohendes Unglück' durch eine Fülle von Signifikanten bezeichnet werden: man träumt von einem Schiffsbruch, einem Sturz vom Pferd, einem Kometen, einem Unwetter, einem bösen Tier etc. Ein Schiff oder Teile desselben hingegen können völlig entgegengesetzte Bedeutungen haben, etwa künftigen Reichtum, eine Reise, den Verlust der Heimat signifizieren, je nach dem narrativen Kontext des Traums. Die Bedeutung richtet sich aber auch z.B. nach dem Geschlecht, dem Alter und Stand des Träumenden. Solche Varianten sowohl auf der Seite des Signifikanten

25 Ebd. S. 11. 
wie des Signifikats machen die lexikalische Gleichung, a bedeutet b' variantenreich, prozesshaft, zeit- und personenabhängig. Es ist schwierig, den semiotischen Prozess des Traums zu zügeln und zu klassifizieren. Als Beispiel für die Varianz der Bedeutung je nach kulturellen und historischem Kontext sei das Beispiel ,Badeszenen im Traum' gewählt:

„Die ganz Alten hielten das Baden nicht für ein übles Vorzeichen; denn öffentliche Bäder waren ihnen noch unbekannt, und man badete in sogenannnten Sitzwannen; die Späteren aber erklärten, als es schon Badeanstalten gab, sowohl das Baden als auch den Anblick einer Badeanstalt für unheilvoll, selbst wenn man kein Bad nahm. Nach ihrer Meinung bedeutet ein öffentliches Bad wegen des herrschenden Lärms Aufregung, wegen des ausströmenden Schweißes Schaden, ferner seelische Angst und Beklemmung, weil Haut und Oberfläche des Körpers im Bad die Farbe wechseln. Noch heutzutage gibt es Leute, die an der alten Auffassung festhalten und die Träume dementsprechend auslegegen; sie sind jedoch im Irrtum und stellen nicht die Erfahrung in Rechnung. Ehedem bedeuten Bäder ganz natürlich etwas Schlimmes, weil die Leute nicht regelmäßig badeten und Badeanstalten in so großer Zahl nicht zur Verfügung hatten, sondern man nur nach Beendigung eines Kriegsdienstes oder nach Überstehen großer Strapazen ein Bad zu nehmen pflegte (Bad und Baden waren für sie also eine Erinnerung an Strapazen oder Krieg).

Heutzutage aber speisen gewöhnlich die einen nicht eher, bevor sie ein Bad genommen, andere tun es auch nach dem Essen; und dann baden sie vor dem Abendessen noch einmal. So ist in unserer Zeit das Baden nichts anderes als ein Mittel zu einem genussreichen Leben. Deshalb ist das Baden in schönen, hellen und gut temperierten Hallen ein gutes Vorzeichen und beschert Gesunden Reichtum und geschäftlichen Erfolg, Kranken Gesundheit; denn Baden ist ein Zeitvertreib der gesunden, sie tun es nicht aus Not oder Zwang."26

Für das Verfahren der Ermittlung von Bedeutungen des,Badens im Traum' fallen hier mehrere Momente auf, die für Artemidor charakteristisch sind: 1 . Bedeutungen hängen vom historischen Index ab: je nachdem, wie die ,ganz Alten', die Späteren und die Heutigen

26 Ebd. S. 75f (Buch I, 64). 
das Baden in ihren Gebräuchen situiert haben, variiert die Bedeutung im Traum. Bei den ,Heutigen' werden diejenigen, die im Schema der Früheren auslegen, von denjenigen unterschieden, die diese Deutung aufgrund von Erfahrung als Irrtum durchschaut haben. 2. Grundsätzlich gibt es ein dipoliges Bewertungsschema: entweder zeigt ein Traumelement ein Übel oder ein Gut an. 3. Es geht überhaupt nicht um das träumende Individuum, sondern um die transpersonale, ,objektive' Bedeutung von Traumelementen. 4. Im Traum sedimentiert sich die, objektive Semantik' der jeweiligen Kulturstufe. Der zivilisatorische Prozess und die von inm regulierten Gebräuche und Werthaltungen indizieren ein Traumelement als gutes oder böses ,Vorzeichen'. 5. Wenn man wie Artemidor davon ausgeht, dass pronoetische Träume die Sprache der zukunftsmächtigen Götter ist, so ist zu bemerken: die Götter sprechen in den Idiolekten der jeweiligen Kulturstufe. 6. Das heißt für den Traumdeuter, dass er ein erfahrener Kultursemiotiker sein sollte. Er muss weniger die Götter als vielmehr die kulturelle Semantik seiner Gesellschaft kennen, um die Bedeutung eines Traumelements $z u$ identifizieren und mit Bewertungsmarkern zu versehen. Daher erklärt sich, warum Artemidor bei seiner Klassifikation nicht mit den Göttern beginnt, auch wenn ihm dies den Vorwurf des "Mangels an Gottesfurcht" einbringen könne.27 Nicht der Sender der Botschaft ist wichtig für deren Entzifferung, sondern der Code, in welchem die Botschaft verschlüsselt ist. Darin ist Artemidor kein schlechter Medientheoretiker.

\section{Kulturelle Semantik des Traums}

Neben dem jeweils herrschenden kulturellen Kontext ist ferner das soziale Profil des Träumers wichtig, um Bedeutungen zu erschließen: „Es ist für den Träumenden und für den Ausleger nützlich, und nicht nur nützlich, sondern geradezu notwendig, dass der Traumdeuter genau weiß, wer der Träumende ist, dass er über dessen Beruf, seine Herkunft, seine Vermögensverhältnisse, seinen Gesundheitszustand und über sein Alter unterrichtet ist."28 Die semantische Varianz eines Traumelements in Abhängigkeit zum Profil des Träumers soll am Beispiel von Tanz-Träumen konkretisiert werden. Hierbei variiert die Bedeutung je nachdem, welchen Status der Träumende hat: ist er

27 Ebd. S. 24f (Buch I, 10).

28 Ebd. S. 24 (Buch I, 10). 
gesund oder krank, ist er reich oder arm, ist er Sklave, Seemann, Gefangener, Frau oder Mann, ist er selbst ein Tänzer, Schauspieler oder Gaukler. Neben diesen sozialindikativen Bedeutungen gibt es aber auch Tanz-Träume, die für jedermann Gutes oder Schlechtes anzeigen. Ferner hängt die Bedeutung nicht nur vom Träumenden, sondern auch von der Tanzart und der Tanzsituation ab bzw. von der Kombination aller drei Momente:

"Im Traum die Vorstellung haben, man tanze daheim im Kreis seiner Angehörigen, ohne dass eine fremde Person anwesend ist oder zuschaut, ist für jedermann ohne Unterschied ein glückliches Vorzeichen. (...) Dagegen ist es für einen kranken Menschen, Mann oder Frau, von übler Vorbedeutung; er wird wegen der vielen Bewegungen beim Tanz um Sinne und Verstand kommen. Ein böses Zeichen ist es ferner für den, der einen Kranken im Haus hat (...). Niemanden, weder einem Gesunden noch einem Kranken, bedeutet es etwas Gutes, zu träumen, dass man in Gegenwart fremder Leute, mögen es viele oder wenige sein, tanzt oder dass man einen seiner Angehörigen tanzen sieht; im ersten Fall wird jemand im Haus sterben, im letzten der tanzende einen großen Skandal erregen. Ein Kind tanzen zu sehen, zeigt an, dass dasselbe taubstumm werden wird, so dass es seine Wünsche durch Zeichen kundtun muss.

Im Theater zu tanzen, geschminkt und in der entsprechenden Kostümierung, und Anerkennung und Beifall zu ernten, prophezeit einem Armen Reichtum, der jedoch nicht bis ins hohe Alter währen wird; denn ein Pantomime spielt auf der Bühne Rollen von Königen und hat viele Diener um sich, nach der Vorstellung aber bleibt er sich wieder allein überlassen. Einem Reichen dagegen zeigt es wegen der vielfachen Verwicklungen in Stücken Aufregungen oder Prozesse an. Sodann ist dieses Gesicht weder für eine reiche noch für eine arme Frau von guter Vorbedeutung; sie werden in große, aufsehenerregende Skandale verwickelt werden. Tanzt ein Sklave, wie und wo auch immer, wird er viele Hiebe beziehen; wer zur See fährt, wird Schiffbruch erleiden oder allein über Bord gehen und kräftig schwimmen müssen; der eine wird unter Schlägen, der andere beim Schwimmen den ganzen Körper in Bewegung setzen. Glück dagegen bringt das Tanzen einem Mann, der in Fesseln schmachtet; denn weil der Körper beim Tanzen beschwingt und gelöst ist, wird jener aller Fesseln ledig werden. Vollführt jemand beim Tanzen Sprünge, wird inn Furcht und Angst befallen, ein Verbrecher wird 
gekreuzigt werden, und zwar wegen der Höhe und wegen des Ausstreckens der Hände. (...)"29

Um die Zukunftsbedeutung des jeweiligen Traumelements zu identifizieren, führt Artemidor eine Art struktural-semantische Faktorenanalyse von erheblicher Komplexität durch. Bedeutungen variieren 1. nach dem Status des Träumers im Lebenszyklus (Alter), 2. nach dem Status in der Gender-Ordnung, 3. nach dem medizinischen Status (krank/gesund; Krankheitsart), 4. nach dem sozialen Status (frei/unfrei; arm/reich; Beruf), 5. nach der Lokalisierung des Tanzes (öffentlich/privat; fremd/vertraut; Theater, Gefängnis...), 6. nach kulturellen Bewertungen (Scham, Skandal), 7. nach der Art des Tanzes (Anstrengung/ Leichtigkeit; Waffentanz; Berufstanz/ Vergnügungstanz, Art der Musikbegleitung...); 8. nach dem physiognomischen Ausdruck des Tanzes (Leichtigkeit bedeutet Befreiung; Ausstrecken der Hände bedeutet Kreuzigung...). Es kann noch viele Faktoren mehr geben, die zu berücksichtigen sind. Doch das Verfahren wird klar: es geht um die sukzessive Eliminierung möglicher Bedeutungen in einem strukturalen Feld von semantischen Dichtomonien (positiv/ negativ), so dass die jeweils zutreffende Bedeutung ermittelt wird. Der Traumdeuter muss die Verzweigungen von Bedeutungsalternativen, die Verteilungen semantischer Kerne im Feld kennen, um in einer Art kartographischer Bestimmung die je zutreffende Signifikanz zu lokalisieren und auf den Träumer zu beziehen. Nach diesem Verfahren entsteht z.B. die Kette: Erwachsener, Mann, gesund, frei, reich, tanzt selbst, öffentlich, Theater $=$ dies bedeutet negativ: Intrigen und Skandale, Aufregungen und Prozesse drohen.

Für uns heute, die wir gewohnt sind, Träume als Spuren einer individuierten Biographie zu lesen, sie nicht futurisch, sondern memorial zu verstehen, mutet es höchst willkürlich an, in der Weise Artemidors jedem visuellen Element des Traums eine eindeutige, orakelnde Bedeutung zuzuweisen, oft sogar ohne Rücksicht auf den immanenten Kontext des Traums. Immerhin wird man zugeben, dass Artemidor bei seiner semiotischen Klassifikation faktorenreich, geregelt und kenntnisreich, also methodisch vorgeht. Der Traumdeuter muss über ein hohes $M a ß$ an empirischem kulturellem Wissen verfügen. Er muss eine Unzahl von Traumerzählungen gehört

29 Ebd. S. 88-90 (Buch I, 76). 
haben, um auch nur ein kurzes Kapitel z.B. über ,geträumtes Tanzen' zu schreiben. Dabei besteht die Absicht auf Vollständigkeit: der Deuter versucht das Feld möglichst gänzlich auszuschreiten, in welchem geträumte Tänze vorkommen. 30

\section{Der Kollaps der Klassifikation}

Das Kapitel ,geträumte Tänze' bildet ein Element in einem großräumigen Klassifikationsschema, das Artemidor, nicht immer plausibel, in Buch I, Kap. 13-82 entfaltet. Die erste Fallgruppe erfasst Träume, die um Schwangerschaft, Geburt und Kindschaft zentriert sind. Dann folgt eine sehr große Gruppe von Träumen, in denen Teile des Körpers leitend sind. Artemidor folgt hier dem Körperschema von Kopf bis Fuß, wobei auffällig ist, wie viele Träume er erfasst, in denen Teile des Kopfes signifikant werden. Als dritte Gruppe führt er Träume an, in denen Körperpraktiken (Tätigkeiten), aber auch Körperverwandlungen zentral sind. Die vierte Gruppe erfasst Träume aus der Sphäre der Unterhaltung und Geselligkeit. Die fünfte Gruppe ist Träumen aus dem Feld der Nutrition gewidmet. Die sechste Gruppe rückt körpernahe Objekte wie Schmuck und Hausgerät ins Zentrum. Die siebte Gruppe stellt die Sexualität in den Mittelpunkt. Angehängt sind zwei Sonderfälle: im Schlaf vom Schlaf träumen; vom Abschied träumen.

Man muss sich vorstellen, was ein Traumdeuter zu bedenken hätte, hätte er alle Körperteile und Körpervollzüge, alle körpernahen Objekte, zudem die kulturellen Semantiken und sozialen Merkmale dieser Traumelemente auf ihre Bedeutungsvarianten hin zu analysieren: schlechthin alles kann signifikativ sein. Man bemerkt sofort, dass ein solches Verfahren nicht daran scheitert, dass es vielleicht falsch ist, sondern überkomplex. Eine infinite Amplifikation von Sinn, eine immer feinere Verästelung in winzige Traumelemente bringt den Traumdeuter in die Lage prinzipieller Überforderung. Er scheitert nicht an der Dunkelheit der Träume, sondern an der schier unbegrenzten Hybridität seines Verfahrens. Mal ausführlich, mal knapp mustert Artemidor so die ,objektive Semantik' der Traumelemente durch, um eine Art historia naturalis, eine Naturgeschichte des Traums zu entwickeln. Die klassifikatorische

30 Vgl. zum vorangegangenen Absatz die gründliche Arbeit von Hahn, István: Traumdeutung und gesellschaftliche Wirklichkeit. Artemidorus Daldianus als sozialgeschichtliche Quelle. Konstanz 1992. 
Ordnung hebelt sich dabei selbst aus. Im Buch II folgt Artemidor folgenden Motivgruppen: Begrüßen, Kleidung, Schmuck, Wetter, Feuerphänomene, Jagd (einschließlich der Tiere), Schiffe/ Schiffsfahrt, agrikulturelle Phänomene (einschließlich der Pflanzen), Flüsse, Gericht, Königtum, politische Institutionen, Krieg und Kampf, ausführliche Kapitel über Götter in Träumen (II; 33-39), Heroen, Treppen, Ärzte, verschiedene Objekte, Tod und Sterben, Geld, Schwalben, Zähne, Flugträume, ein Kapitel über die Glaubwürdigkeit wahrsagender Berufsgruppen (II, 69), Zahlen in Träumen. Im Buch III folgen ohne jede Ordnung 63 Einzelmotive, in absonderlicher Reihung: so folgt dem Traummotiv, Gott zu werden, ein Abschnitt über Stelzen; dem Wiesel folgt der Lehm; der Hebamme die Diestel; dem Gras die Bewachung; den Grillen die Zwiebel etc. Die Bücher IV und $V$ sind nicht mehr an den Freund Cassius Maximus adressiert, sondern an den eigenen Sohn. Sie stellen Ausführungen über Methodiken der Traumauslegungen und die Exegese verschiedenster Traumotive willkürlich nebeneinander. Im Buch $V$ folgen überwiegend Traumsplitter und Einzelträume; abrupt bricht das Buch und damit das Gesamtwerk ab.

Die zunächst versuchte Architektur des Traums ruiniert zunehmend unter der wuchernden Fülle disparater Einzelheiten, die den Bau des Buchs zersetzen und die Ordnung vollends zerfransen lassen. Am Traumbuch des Artemidor zeigt sich exemplarisch, was die Crux aller naturgeschichtlichen Tableaus bis ins 18. Jahrhundert sein wird: sie werden uferlos. Was Ordnung erzeugen soll, wird zur Ursache des Chaos - nämlich die enzyklopädische Anstrengung. Es zeigt sich, dass die Einteilung nach Motiven oder Objektklassen überhaupt ungeeignet ist, den Traumprozess als solchen zu konstruieren. Dagegen sind die einzelnen Träume, insbesondere wenn sie zu kleinen novellistischen Miniaturen gerinnen, spannend und das Traumbuch ist hinsichtlich der darin aufbewahrten kulturgeschichtlichen Einzelmomente höchst aufschlussreich. Unter der Hand aber verwandelt sich das Vorhaben, ein Weltbuch des Traums zu schreiben, in ein wüstes Sammelsurium von Traumfragmenten, zu einer gewaltigen Halde von signifikativen Zeichen, die - so viele sie sein mögen - doch geringfügig sind im Verhältnis zu dem, was im Traum bedeutend werden kann, nämlich alles. Artemidor benötigte ein unendliches Buch, um mit seinem Verfahren die Traumwelt zu erschöpfen. Es ist aussichtslos, die Dinge 
der Welt oder die Dinge des Traums klassifizieren zu wollen: die Klassifikation bricht zusammen und der Aufzählung ist kein Ende.

Nichts macht die epochale Wendung, die Freud vollzogen hat und von der Adorno spricht (s. Motto)31, deutlicher, als dass Freud nur noch Reste motivlicher Klassifikationen aufweist; statt dessen konzentriert er sich auf die Prozessmechanismen des Traums. Diese Komplexitätsreduktion, durch die an Stelle eines unendlichen Archivs der Lakonismus von acht Traummechanismen tritt, aus denen das Universum aller Träume generiert werden kann -: dies ist die Wendung, die Freud in der Geschichte der Traumdeutung herbeiführt. Bei Artemidor haben wir, in symptomatischer Wirrnis, das Phantasma eines Museums der Träume, die verzeichnet, benannt, in Reihen gebracht, archiviert und deponiert werden sollen. Doch die Verzeichnisse beginnen sinnvoll und enden absurd. Die Benennungen wechseln willkürlich zwischen Einzeldingen, Klassen, Handlungsarten. Die Reihen brechen abrupt ab, tauchen irgendwo wieder auf, springen, mäandern, überkreuzen sich in verwilderten Texturen. Das Archiv, das ordentlich begonnen wird, wird hybrid und vom niemals endenden Nachschub der Traumarchivalien schließlich vollgestopft. Es fehlt jede sinnvolle Registratur, jeder Code oder Schlüssel, so dass das Traumarchiv zum Labyrinth mutiert, dessen konstruktive Form verloren ist, ein verworrenes Depot, das gleichzeitig an Überfülle und Leere leidet.

Man erinnert sich an die Erinnerung Michel Foucaults, als er eingangs der "Ordnung der Dinge" Jorge Luis Borges zitiert, der sich an eine gewisse chinesische Enzyklopädie erinnert, in welcher die Tiere in Klassen unterteilt werden, die unter keiner Bedingung rational nachvollziehbar, also absurd und grotesk sind - und vielleicht deswegen der Heterogenität der Phänomene nahe kommen.32 Dies trifft auch für die Taxinomie des Traums bei Artemidor zu. Sie wäre

31 Diese Wendung setzt freilich in mancher Beziehung schon in der frühen Neuzeit ein; vgl. dazu Niessen, Stefan: Traum und Realität - ihre neuzeitliche Trennung; Würzburg 1993. - Schirrmeister, Albert: Rationalität und Geschichte. Zum Status von geträumter Wahrnehmung in spezifischen kulturellen Kontexten der Frühen Neuzeit. In: Karafyllis, Nicole C./Schmidt, Jan C. (Hg.): Zugänge zur Rationalität der Zukunft; Stuttgart 2002, S. 99-115. - Ferner der schöne Katalog von Lynn Gamwell (Hrsg.): Träume 1900-2000. Kunst, Wissenschaft und das Unbewusste. Wien 2000.

32 Foucault, Michel: Die Ordnung der Dinge. Eine Archäologie der Humanwissenschaften; 3. Aufl. Frankfurt/M. 1980, S. 17. 
nur grotesk, wenn sie nicht zugleich grundlegende Probleme des Traumverstehens zum Ausdruck brächte:

1. Traumsammler der Art Artemidors sind Besessene des Details, der überdeterminierten Einzelheit, des winzigen Fragments. Sie betreiben eine Art Mikrologie, eine Elemenentarteilchen-Semantik des Traums. Und dies macht ihre Texte zu einem Kryptogramm, zu einer labyrinthischen Rätselschrift des Sonderlichen, Kuriosen und Monströsen.

2. Da Traumdeuter wie Artemidor - ähnlich wie Naturhistoriker alles auflesen, ohne die dynamischen Mechanismen der Traumproduktion zu kennen, kleben sie an der unendlichen Masse der Traumprodukte, die sie zu einer Art Naturalienkabinett des Traums versammeln.

Niemand kann sich darin orientieren. Obwohl Artemidor in seiner Einleitung (Buch I, 1-13) mehrere Klassifikationsschemata vorstellt und dem entsprechend methodisch vorgeht, schlägt seine Darstellung in Chaos um: Niemand bis heute war vermögend, das Buch Artemidors wenigstens nachträglich zu organisieren. Es ist unmöglich für uns, um wieviel mehr für seine Adressaten, Freund und Sohn, denen er doch den Leitfaden einer wissenschaftlichen und methodisch sicheren Traumdeutung an die Hand geben wollte.

\section{Kulturelle und divinatorische Hermeneutik}

Doch durch die obsessionelle Aufmerksamkeit auf jedes Detail wird Artemidors Buch, gewiss unfreiwillig, zu einer Fundgrube, einem Kramladen antiker Praktiken und Gebräuche, von umlaufendem Aberglauben, seltsamen Deutungen und merkwürdigen Einzelheiten des Alltags. Gewiss ist es ausgeschlossen, die Traumbedeutung von Insekten erfassen und auflisten zu wollen; aber man erfährt viel über das Verhältnis antiker Kultur zu Ungeziefer und Insekten. In diesem Sinn werden bei Artemidor die Traumtexte zu verrückten, phantasmagorischen Umschriften antiker Alltagskultur. Die Traumwelt verwandelt sich zu einem musée imaginaire der Gesellschaft und als solches wird der Text aufschlussreich und spannend. Er überliefert, im Vermeinen, die ,objektiven Bedeutungen' göttlicher Botschaften zu entziffern, eine ungeheure Menge kultureller Fragmente, die das Puzzle eines verlorenen Volksglaubens ergeben. Dadurch werden die Oneirokritika zu einer wichtigen kulturgeschichtlichen Quelle. Dies geschieht nicht versehentlich. Denn für Artemidor ist die Kenntnis kultureller Kontexte eine Voraussetzung der richtigen Traumdeutung: 
"Falls du", so dekretiert er, "über örtliche Sitten und Gebräuche und über Land und Leute nicht Bescheid weißt, so informiere dich. Reisen und Belesenheit werden dir am ehesten ein Wissen darüber verschaffen; denn Bücher über Traumdeutung allein reichen nicht aus, um dich zu fördern, es müssen alle anderen Wissensgebiete dazukommen." 33

Zum Traumexperten wird also erst der ethnographisch erfahrene und enzyklopädisch Gebildete. Weil im Traum alles zum Zeichen wird, muss der Traumdeuter alles wissen: gerade dieser Maximalanspruch, der selbst ein Phantasma ist, erzeugt eine Überlastung, die notwendig das Chaos nach sich zieht. Die eigentümliche Mischung aus methodischer Ordnung und phantastischem Chaos scheint Artemidor gelegentlich selbst zu spüren - um diesen Anflug des Zweifels gleich wieder wegzuwischen: "Man mache sich einmal klar, dass nichts so schwierig und mühevoll ist, als die Verschlingung und Verdichtung der Traumbilder zu durchschauen und alle in einer einzigen Aussage zusammenzufassen, weil sie vielfach einander widersprechen und nichts gemeinsames haben. Es ist aber schlechthin ausgeschlossen, dass die gegebenen Zeichen im Widerspruch zueinander stehen, wenn je die Traumgesichte alles, was sich in Zukunft ereignet, vorhersagen. Vielmehr, wie es im Tagesgeschehen eine Ordnung und eine Reihenfolge gibt, so müssen auch die Traumgesichte einer gewissen Ordnung unterliegen." 34 Artemidor kann das Verschlungene seines Textes, wuchernden Tropen, nur durch einen verzweifelten dogmatischen Sprung retten: durch das Dekret, dass Unordnung, so sehr sie sich dem Eindruck des Lesers aufdrängt, "ausgeschlossen" ist. Die Unordnung des Textes ist nur der Schein, dem der Uneingeweihte unterliegt. "Die Traumgesichte" sind "infolge der Verdichtung der in ihnen auftauchenden Zeichen vieldeutig und für die meisten unergründbar".35 Der Kenner indes wird "bei allen vielschichtigen Traumgesichten die Deutungen herauszufinden" verstehen, "indem man jedes einzelne Kernstück zu einem abgerundeten Ganzen fügt und verschmilzt". ${ }^{36}$ Was wie eine Archäologie des Traums aussieht, durch die man das Relikt zum Kern

33 Artemidor (wie Anm. 16), S. 259 (Buch IV, 4).

34 Ebd. S. $240 f$ (Buch III, 66).

35 Ebd.

36 Ebd. S. 242 (Buch III, 66). 
eines Gesamtgefüges zu bilden versteht, ist von Artemidor indes als Parallele zu hieratischen Verfahren zu verstehen: für den Uneingeweihten sprechen auch die Träume in fragmentierten Rätselbildern, doch fügt sie der Priester zu einem Ganzen: „Man muß seine Auslegungen nach Art der Opferpriester geben, die einerseits genau wissen, wohin jedes einzelne Zeichen passt, andererseits ihre Urteile ebenso sehr aus jedem einzelnen als aus allen Zeichen zusammen schöpfen." 37

Aufschlussreich ist, dass die hermeneutische Schließung zu einem Ganzen hier als Gegenmaßnahme zum Selbstverslust des Deuters in der Fülle der inkompossiblen Einzelheiten und der widersprüchlichen Chaotik der Phänomene aufgeboten wird. Daraus erwachsen die Orakelurteile, welche die verworrene Sprache der Dinge durch Divination in ein ,gefügtes' Ganzes verwandeln.

Es ist deutlich, dass der von Traummotiven ausgehende Ansatz eine unbeherrschbare Komplexität erzeugt, die der Deuter nur dogmatisch und durch eine divinatorische Hermeneutik zügeln kann. Gleichwohl versteht Artemidor sein Vorgehen ausdrücklich als wissenschaftlich und legt größten Wert auf plausible Begründungen von Traumexegesen. Er steht auf der Schwelle zwischen Priester und profanem Experten. Das Scheitern seines Ansatzes ist weniger darin begründet, dass die Menschen nicht mehr an Träume als Orakel glaubten, sondern dass das Wuchern der Zeichen eine Überfülle erzeugt, an der die Traumdeutung erstickt. Im Verhältnis zu solchen, bis weit in die Neuzeit herrschenden Modellen ist es eine geniale Vereinfachung, wenn Freud fast völlig darauf verzichtet, ein Motivlexikon von Traumelementen aufzubauen, sondern die Aufmerksamkeit vom Produkt auf die Produktion des Träumens umstellt. Der Deuter muss jetzt kein unendliches Archiv von Traumsymbolen lernen, sondern wissen, nach welchen Regeln der Traum arbeitet. Dies ist eine Frage, die Artemidor nur ganz am Rande interessiert, wenn er z.B. die Analogie oder die Verdichtung als Traummechanismus benennt. Geht man derart vom Produzieren und nicht vom Produkt aus, kann es keine Vorab-Bestimmungen von Bedeutungen geben - also kein Traumlexikon. Mit Freud wird der Traumhermeneut davon befreit sein, ein divinatorischer Chiffrenkundiger objektiver Bedeutungen zu sein; statt dessen wird 
er jeden einzelnen Traum daraufhin untersuchen, durch welche Mechanismen er seine Erscheinungsoberfläche erhalten hat. Daraus werden die Richtungen abgeleitet, in welcher der ganz individuelle Sinn eines Traums gesucht werden muss.

Die Wendung Freuds ist indes nicht nur ein Gewinn, sondern auch ein Verlust: fortan träumen die Menschen nur noch im privaten Gehäuse ihrer Wünsche und Affekte. Während bei Artemidor das Träumen die Seele mit den Göttern und mit der Zukunft in Korrespondenz setzt: durch das Nadelöhr der träumenden Einbildungskraft geht das ganze Universum und das Ganze der Kultur hindurch.

\section{Ludwigs Binswangers "Traum und Existenz"}

Der Psychiater und Philosoph Ludwig Binswanger geht in der vom jungen Michel Foucault kommentierten Arbeit "Traum und Existenz" (1930/47) einen Weg, der die Individualisierung des Traums bei Freud und die ,objektive Semantik' Artemidors verbindet. Der Traum vermittelt durchaus das Objektive, das unhintergehbar das Individuum angeht und betrifft: so die Ausgangsthese. Aus den sprachlichen, dichterischen und traumhaften Befunden leitet Binswanger eine Art doppelte Matrix ab: das Steigen und das Sinken, das Erhobenwerden, Fliegen, Schweben und das Fallen, Stürzen, Straucheln, Schwerwerden. Diese Richtungspolarität ist sicherlich schon mit der Leiblichkeit und den an diese gebundenen Gefühlen gegeben. Aber der Leib ist für Binswanger keine Basis, von der her die Doppelmatrix des Steigens und Sinkens abgeleitet werden könnte. Denn diese Doppelmatrix erscheint nicht zufällig auf allen Ebenen seelischer und geistiger Aktivität, der sprachlichen Semantiken, der ,Gerichtetheit' ganzer Weltanschauungen oder Kulturen: darin spiegelt sich mehr als nur leibliche Schwere und Leichtigkeit, sondern eben - ,Objektives'. Die Richtungspolarität des Steigens und Sinkens codiert für Binswanger grundlegende "Bedeutungsrichtungen", und zwar in dem strikten Sinn, dass darin ein "ontologisches Existenzial" erlebt, gefühlt, gedacht, gespürt, geträumt oder symbolisiert wird. Die Richtungs- und Bedeutungsräumlichkeit ist selbst nicht weiter ableitbar, sondern sie ist umgekehrt ein generatives Schema, in das wachende wie träumende Aktivitäten eingebettet sind. Immer gilt, dass das ,Ich' "das Ursubjekt dessen bleibt, "das steigt und fällt". Es ist immer Ich, der im Steigen oder Fallen begriffen ist. Doch ist dies nichts 
Individuelles, sondern eine objektive Struktur, ein Rhythmus der transpersonalen Lebensbewegung, die das ,Ich' immer schon und je wieder ist und die doch durch das Ich hindurch und über es hinausgeht. Immer wenn - in einem plötzlichen Affekt, einem poetischen Bild, einer empathischen Wahrnehmung - dieses Steigen und Sinken sich in unausweichlicher Evidenz aufdrängt, bin ,ich' ganz ,da'. Binswanger stellt fest, dass viele Träume von diesen Bedeutungsrichtungen strukturiert werden und die "Gestimmtheit" des Traum angeben. In dieser Weise sind Träume Anzeiger eines existenzialen Grundtonus, der den Träumenden bestimmt - sei es, dass seine Kräfte zu frischem Impuls Auftrieb geben, sei es, dass niederdrückende Momente die Intentionen schwer machen und zu Boden drücken. Steigen und Sinken, Fliegen und Stürzen sind demnach allgemeinste Grundrichtungen, durch die das Ich sich selbst gegeben ist. Es gibt das Ich, insofern es steigt und sinkt. Damit ist die anticartesianische Behauptung aufgestellt, dass das "Ich bin" nicht durch Akte des Bewusstseins gegeben wird. Sondern das Allerpersönlichste des Ich ist eine Gabe der allgemeinsten Bewegungsrichtung, der Richtungsräumlichkeit. Schärfer gesagt: das Ich ist eine Funktion des Raumes bzw. der räumlichen Bewegungsfiguren, die zu Existenzialien des Egos erhoben werden.

Von daher muss sich Binswanger nicht wie Artemidor in mikrologischer Detailversessenheit auf die Bedeutungsrätsel kleinster Traumelemente konzentrieren. Und er muss auch nicht wie Freud die Erscheinungsoberfläche des Traums durchstoßen, um den lebensgeschichtlichen Urtext dieses besonderen Individual-Ichs zu treffen. Sondern Binswanger muss am Traum den Grundtonus, die Grundrichtung des Träumers identifizieren, um die ebenso überindividuelle wie jeweilige Gestimmtheit des träumenden Ich zu erkennen. Ihn interessieren nicht einzelne Bedeutungen, sondern die dominante Gerichtetheit der Existenz, in welche dann alle einzelnen Traumelemente eingetaucht und semantisch codiert sind. So kann man sagen, dass der Traum bei Binswanger ebenso objektiv wie subjektiv ist, Artemidor wie Freud.

Wenn es aber weder das einzelne Ich ist, das träumt, noch die Götter den Traum ,objektiv' eingeben: wer ist es dann, der bei Binswanger träumt? $\mathrm{Er}$ holt weit aus. Von Petronius, dem freigeistigen Zeitgenossen Neros, zitiert er: "Die Träume, die den Geist spielerisch mit willkürlichen Schatten erfüllen, sind weder 
göttliche Eingabe, noch werden sie als numinose Botschaften aus dem Äther geschickt; sondern ein jeder macht sich die Träume selbst." Man erkennt den Widerspruch gegen die orakelnde Traumauffassung à la Artemidor. Dennoch zögert Binswanger, bei Petronius schon die erste Spur einer individualpsychologischen Traumauffassung auszumachen. Er fragt sich vielmehr: wer ist dieser "ein jeder" (quisque), der sich seine Träume selbst macht?38 Binswanger zeigt, das der ,Jeder' eine abwertende, "doxische Form" ist, eine Schwundstufe gegenüber der objektiven Form der Gemeinschaftlichkeit (logos), ein "gänzlich unbestimmtes X gleichsam hinter dem Traum". Es scheint, dass Binswanger den heraklitischen Gegensatz von gemeinschaftlicher Tagwelt des Logos und idiosynkratischer Nachtwelt des Traums zu unterlaufen gedenkt.

Denn die Deutung der Träume als bloß privates Kopfkino, als bloße doxa, wiederspricht der Binswangerschen Konzeption, worin "der überindividuelle Bildgehalt" des Traums "zwar nicht von jedem einzelnen gemacht" wird, "wohl aber hat inn jeder einzelne im Traum. (...) seine Bilder $(\ldots)$ gehören inm allein an, er lebt ganz und gar in seiner eigenen Welt". Dies erinnert an den ídios kósmos bei Heraklit. Dennoch aber seien die Bildgehalte nicht private Willkür (bloße doxa), sondern überindividuell. Was soll das heißen?

Mit Hegel anerkennt Binswanger, dass das Träumen den Träumer aus dem Zusammenhang des Ganzen und Gemeinsamen herausschneidet. Der Träumer ist, nach Hegel, „Bewusstsein ... der Einzelheit... Eine Originalität, die eine Eigentümlichkeit (idiásmen, HB) des Inhalts und der Form wird, ist das Unwahre und Schlechte." Traum ist bei Petronius wie Hegel, auf der Spur Heraklits, die radikale "Vereinzelung des Denkens". "Das Träumen ist ein Wissen von etwas, wovon nur ich weiß," nämlich "die Weise, daß etwas bloß für mich ist, ich etwas in mir, als in diesem Subjekte, habe". 39 Dieser Quisque ist eine schlechte Subjektivität, die sich nicht entfaltet, entäußert und durchgearbeitet hat, als unfrei ist: so Hegel. Traum in diesem Sinn, wie alles Imaginäre, ist dann bloße "Privatheit".

38 Binswanger, Ludwig: Traum und Existenz. Aus dem Französischen und mit einem Nachwort von Walter Seitter; Bern - Berlin 1992, S. 124.

39 Ebd. S. 129. Die Hegel-Zitate nach Hegel, Georg Wilhelm Friedrich: Vorlesungen über die Geschichte der Philosophie I. In: Werke in zwanzig Bänden; Frankfurt am Main 1995, Bd. 18, S. 342. 
Dagegen will Binswanger den Traum aufwerten. Wenn er am Traum dasjenige betont, was eine überindividuelle Struktur, eben die Richtungsräumlichkeit codiert, die genau dieses Ich betrifft und doch ein Allgemeines an inm hervorkehrt, dann ist der Traum nicht nur eine Hegelsche "Beschränkung" auf die originale, aber unfreie Einzelheit. Sondern im Gegenteil reißt der Traum das Ich aus seiner Verfallenheit an die Unruhe, Rastlosigkeit, Verstrickung privater täglicher Lebenstätigkeiten, befreit das Ich aus der "Leidenschaft der Innerlichkeit" (Kierkegaard). Der Traum enthält zwar Bilder dieser wimmelnden Unruhe, doch kommt es darauf an, den darin anklingenden Grundton zu vernehmen, das spezifische Sinken und Steigen, um dadurch "Beziehung auf ein Allgemeines" zu gewinnen. Das ebenso willkürliche wie wirre Spiel der Schatten, von dem Petronius spricht, enthält einen Grundcode, der das radikal Einzelne des Traums - seine Eigenwelt - mit der transpersonalen gemeinschaftlichen Welt verbindet und gerade von den "falschen Hüllen der Persona einerseits und der Suggestivwelt unbewusster Bilder andererseits" befreit.40 Der Traum wird hier als Möglichkeit entworfen, das Ich aus der Verfallenheit an das "Man" und das "Gerede" der öffentlichen Welt (Heidegger) zurückzuholen und vor sich selbst zu bringen. Man bemerkt die Nähe zur Existenzialphilosophie der 20er und 30er Jahre. Sie erhält hier die besondere Wendung, dass es gerade der Traum ist, der das Ich vor sich selbst bringt, und zwar in seinem Überindividuellen. Mit Heidegger sagt Binswanger, dass solcherart im Traum "das Dasein vor sein Sein gebracht wird", "insofern als ihm (= dem Träumenden) etwas geschieht, und ... er nicht weiß, wie und was inm geschieht. Das ist der ontologische Grundzug alles Träumens und seiner Verwandtschaft mit der Angst! Träumen heißt: Ich weiß nicht, wie mir geschieht. "41 Diese ,Ich-Mir' Relation im Geschehen des Traums heißt, dass das Selbst vor das Ich gebracht wird. Das Selbst aber ist "eine rein formale Anzeige ohne Substanz, der Spielball des steigenden und fallenden Lebens". Dieses unpersönliche Selbst - der Quisque -, wird vor das Ich gebracht und vor die Möglichkeit gestellt, seine eigene Lebensform zu ergreifen bzw. sich zu dieser zu entscheiden. Das wäre die existenziale Möglichkeit des Traums, in welchem die Willkür

\footnotetext{
40 Ebd. S. $132 / 3$.

41 Ebd. S. 134.
} 
der Bilder als Anzeige des eigenen Seinkönnens verstanden wird. Das nimmt die pronoetische Funktion der Träume bei Artemidor auf, nicht etwa die memoriale Form Freuds.

Man erkennt, wie sehr auch Binswanger dem Traum die Lesart seiner Zeit unterschiebt. Artemidor, Freud, Binswanger - sie alle zeigen, dass das Geschehen des Traums niemals verstanden werden kann, ohne es zu konstruieren; wodurch das Geschehen aufhört, Ereignis zu sein, und der Traum notwendig in eine Konstruktion des Wachbewusstseins verwandelt wird. Wenn wir den Traum zum Wissen des Allgemeinen machen, ist der Traum schon nicht mehr. Nach Hegel ist das Wissen, wovon nur ich weiß, selbst ein Träumen das bloß für mich ist. Man kann, mit anderen Worten, den Traum nur träumen. Das aber ist nur ein idiosynkratisches Wissen. Wer mehr vom Träumen will - wie alle Traumdeuter -, der steht schon jenseits des Traums, nämlich in der Wachwelt des Bewusstseins und der Allgemeinheit - und schreibt dieses in den Traum hinein. Je näher wir dem Traum mit unseren Deutungen rücken, umso mehr flieht er. Aus diesem Dilemma befreit keine Traumdeutung. Aufklärend zu deuten, haben wir, nach einem Wort Robert Musils, Vernunft gewonnen, aber Traum verloren. 